\title{
What are the effects of macroalgal blooms on the structure and functioning of marine ecosystems? A systematic review protocol
}

\author{
Devin A Lyons ${ }^{1 *}$, Rebecca C Mant ${ }^{2}$, Fabio Bulleri ${ }^{3}$, Jonne Kotta ${ }^{4}$, Gil Rilov ${ }^{5}$ and Tasman P Crowe ${ }^{6}$
}

\begin{abstract}
Background: Anthropogenic activities are believed to have caused an increase in the magnitude, frequency, and extent of macroalgal blooms in marine and estuarine environments. These blooms may contribute to declines in seagrasses and non-blooming macroalgal beds, increasing hypoxia, and reductions in the diversity of benthic invertebrates. However, they may also provide other marine organisms with food and habitat, increase secondary production, and reduce eutrophication. The objective of this systematic review will be to quantify the positive and negative impacts of anthropogenically induced macroalgal blooms in order to determine their effects on ecosystem structure and functioning, and to identify factors that cause their effects to vary.

Methods: We will search a number of online databases to gather empirical evidence from the literature on the impacts of macroalgal blooms on: (1) species richness and other univariate measures of biodiversity; (2) productivity and abundance of algae, plants, and animals; and (3) biogeochemical cycling and other flows of energy and materials, including trophic interactions and cross-ecosystem subsidies. Data from relevant studies will be extracted and used in a random effects meta-analysis in order to estimate the average effect of macroalgal blooms on each response of interest. Where possible, sub-group analyses will be conducted in order to evaluate how the effects of macroalgal blooms vary according to: (1) which part of the ecosystem is being studied (e.g. which habitat type, taxonomic group, or trophic level); (2) the size of blooms; (3) the region in which blooms occurred; (4) background levels of ecosystem productivity; (5) physical and chemical conditions; (6) aspects of study design and quality (e.g. lab vs. field, experimental vs. observational, degree of replication); and (7) whether the blooms are believed to be anthropogenically induced or not.
\end{abstract}

\section{Background}

Coastal marine ecosystems are under increasing pressure from multiple drivers of human-induced environmental change, including resource extraction, habitat modification and destruction, species introductions, ocean acidification, and inputs of pollutants and nutrients [1]. One of the consequent changes experienced by these ecosystems is an increase in "species outbreaks", or large, rapid, and temporary increases in the abundance of a species. These outbreaks can, in turn, cause changes in the structure and function of the broader ecological community, modify the physical and chemical environment, and affect human health, ecosystem services, and the economy [2-5].

\footnotetext{
* Correspondence: devin.lyons@ucd.ie

'Lead reviewer, University College Dublin, Dublin, Ireland

Full list of author information is available at the end of the article
}

Large blooms of macroalgae provide a striking example of species outbreaks. These blooms are normally formed by opportunistic, ephemeral algae, which are a natural component of shallow-water marine communities [6]. However, humans are believed to have increased the magnitude, extent, frequency, and duration of their proliferation by increasing nutrient loads in coastal waters and inducing trophic cascades that release seaweeds from herbivory $[5,7]$.

The effects of macroalgal blooms are varied, with social, economic and ecological dimensions. Blooming macroalgae interfere with recreation and aesthetic enjoyment of coastal areas, while toxins released from decomposing algae pose a threat to the health of humans and domestic animals $[8,9]$. As a result, macroalgal blooms have the potential to reduce income from tourism and necessitate costly algal removal programs. The ecological effects of
C Biomed Central 
macroalgal blooms are generally seen to be negative. Macroalgal outbreaks have been linked to declines in the abundance of seagrasses and non-blooming macroalgae $[10,11]$. Decaying blooms can contribute to hypoxia and increased free sulphide in sediments and surrounding waters, thereby reducing the abundance and diversity benthic invertebrates [12-15]. However, the effects of macroalgal blooms are not uniformly negative. Algal accumulations can increase habitat complexity, provide tolerant species with food or shelter, and enhance both dispersal and secondary production [16-19]. The negative effects of macroalgal blooms on seagrasses may not occur unless herbivory is intense [20]. Macroalgal blooms may even help to reduce the eutrophication that causes them. They transfer nutrients from eutrophic waters to the sediments and other macroalgae, thereby reducing nutrient uptake by phytoplankton positive feedbacks to further eutrophication [21,22].

Given the varied positive and negative ecological effects of macroalgal blooms, quantifying their overall effects on ecosystem structure and functioning, and identifying the factors that determine how and why these effects vary are an important challenge. Meeting this objective would enable us to obtain more complete estimates of their effects on ecosystem services and the economy. Finally, it would provide relevant authorities with evidence-based knowledge that will facilitate decisions about how to best manage macroalgal blooms and the anthropogenic drivers that cause them.

\section{Objective of the review}

This review will synthesize the evidence for effects of ephemeral macroalgal blooms on the organization and dynamics of marine and estuarine ecosystems. The focus will be on quantifying effects that may influence the services that these ecosystems provide, and the economic activity that they generate. This review will have a global geographic scope. However, it will not address the wellstudied effects of macroalgae on coral reefs, which have been extensively reviewed [e.g. 23-25], synthesized, and evaluated in a recent meta-analysis [26]. The primary research question is:

What are the effects of macroalgal blooms on the structure and functioning of marine ecosystems?

Ecosystem structure and ecosystem functioning are complex concepts, with varying definitions. In quantifying the effects of macroalgal blooms on ecosystem structure, we will focus on how the diversity and evenness of the biological communities within the ecosystem are affected, as well as the abundance of other primary producers (e.g. seagrasses, other algae), fish, invertebrates, and birds. In quantifying the effects on ecosystem functioning, we will focus on functions such as: the productivity of other primary producers; secondary productivity; biogeochemical cycling of carbon, nitrogen, phosphorus, oxygen, and sulphur; and other flows of energy or material (e.g. subsidies to other ecosystems, trophic interactions). In assessing the evidence for these effects, we intend to address seven secondary questions:

1. Do different components of the ecosystem (e.g. particular trophic levels, functional groups or taxonomic groups) respond differently to macroalgal outbreaks?

2. How do the effects of outbreaks vary with their magnitude, duration and spatial extent, or the duration and extent of the studies examining them?

3. How do the effects differ in different regions (i.e. "large marine ecosystems")?

4. How do the effects vary between habitat types (e.g. pelagic vs. benthos, subtidal vs. intertidal, estuary vs. ocean)

5. Do the effects differ in areas of differing productivity?

6. Do the effects vary with environmental variables, such as temperature, water flow, and nutrient concentrations?

7. Do blooms attributed to anthropogenic causes have different effects from those that are naturally occurring?

\section{Methods}

\section{Search strategy}

We will search for relevant studies using Web of Science and Scopus databases, using the search terms listed below. The search terms for macroalgal blooms will be combined with the other terms using the Boolean operator 'AND'. The search terms for ecosystem structure, biogeochemical cycling, and productivity will be separated using the Boolean operator 'OR' and then combined within a set of outer brackets.

\section{Terms for macroalgal blooms}

(((Enteromorpha OR Ulva OR ulvoid OR Ulvaria OR Gracilaria OR "Pilayella littoralis" OR Cladophora OR Chaetomorpha OR Pylaiella OR Ectocarpus OR Lobophora OR wrack) AND (bloom* OR outbreak OR proliferation OR overabundance)) OR "green tide" OR "macroalgal bloom" OR "seaweed bloom"" OR "macroalgal accumulation" OR "accumulation* of macroalga*" OR "alga* mat" OR "alga* mats" OR "macroalgal mat" OR "accumulation* of algae" OR "drifting alga" OR "drifting macroalg*" OR "nuisance macroalga*" OR "ephemeral alga*" OR "opportunistic alga*" OR (("Alga* bloom*" OR "alga* outbreak" OR "Nuisance alga") AND (wrack OR seaweed OR macroalga* OR Enteromorpha OR Ulva OR Ulvaria OR Gracilaria OR Pilayella OR Cladophora OR Chaetomorpha OR Pylaiella OR Ectocarpus OR Lobophora))). 


\section{Terms for ecosystem structure}

(hysteresis OR "tipping point" OR "alternat" state" OR "alternat" stable state" OR "phase shift" OR "regime shift" OR "species richness" OR diversity OR "community structure" OR evenness OR "Shannon-Weaver" OR "ShannonWeiner" OR "Shannon index" OR "Simpson Index" OR "abundance-biomass curve*" OR "species abundance distribution*").

\section{Terms for biogeochemical cycling, flows of energy and materials}

("Energy flow*" OR "Energy flux*" OR "Flow* of energy" OR "Flux" of energy" OR biogeochemical OR "Nutrient cycl" OR "cycling of nutrient" OR "nutrient flux" OR "Nutrient flow*" OR "Flow" of nutrient*" OR "Flux* of nutrient*" OR "cycling of carbon" OR "carbon cycl*" OR "carbon stor*" OR "carbon flow*" OR "carbon flux" ${ }^{*}$ " OR "flow" of carbon" OR "flux* of carbon" OR "cycling of sul"ur" OR "Sul"ur cycl*" OR "Flow* of sul"ur" OR "flux* of sul"ur" OR "sul"ur flux*" OR "sul"ur flow*" OR "Hydrogen sul*ide" OR "cycling of nitrogen" OR "nitrogen cycl*" OR "Flow* of nitrogen" OR "flux* of nitrogen" OR "nitrogen flux*" OR "nitrogen flow*" OR denitrification OR "cycling of phosphorus" OR " phosphorus cycl"" OR "Flow* of phosphorus" OR "flux* of phosphorus" OR "phosphorus flux" ${ }^{*}$ OR "phosphorus flow*" OR "cycling of oxygen" OR " oxygen cycl*" OR "Flow" of oxygen" OR "flux* of oxygen" OR "oxygen flux*" OR "oxygen flow*" OR anoxi* OR hypoxi* OR bioturbation OR grazing OR foraging OR herbivory OR predation).

\section{Terms for productivity and abundance}

("primary product*" OR "secondary product*" OR "carbon fixation" OR "community respiration" OR "ecosystem respiration" OR "community metabolism" OR "ecosystem metabolism" OR "abundance of benthic" OR "productivity of benthic" OR "benthic biomass" OR "biomass of benthic" OR "benthic metabolism" OR "benthic respiration" OR ((abundance* OR biomass* OR productivity OR mortalit* OR survival OR growth OR cover OR densit*) AND (fauna* OR animal OR infauna* OR epifauna* OR fish OR macroinvertebrate OR invertebrate OR macrofauna* OR mesofauna* OR meiofauna* OR epibenthic OR seagrass OR eelgrass OR Cymodocea OR Zostera OR Posidonia OR seaweed OR macroalgal OR macroalgae OR fish* OR bird* OR seabird OR shorebird))).

\section{Study inclusion criteria}

We will evaluate studies for inclusion at three successive levels, based on whether their populations, interventions, comparators, outcomes, and study types are considered relevant (see inclusion criteria below). First we will evaluate the titles, and remove spurious citations. Next, we will evaluate the abstracts. Several members of the review team will independently assess a subset of the studies ( 10\%), and a multi-rater Kappa statistic relating to the assessments will be calculated. If the statistic indicates that reviewers are inconsistent in their assessment $(\mid<0.5)$, discrepancies will be discussed and the inclusion criteria will be clarified or modified. Finally, we will evaluate the remaining studies at full text. If it is not clear whether a study meets our inclusion criteria at one of the levels of screening, it will be evaluated at the next level. The inclusion criteria we will use are as follows:

\section{Relevant populations}

Any ecosystems or ecosystem component (excluding coral reefs) affected by marine or brackish-water macroalgal blooms, including but not limited to:

- Coastal, estuarine, and lagoon ecosystems

- Seagrasses, algae, invertebrates, fish, and birds

- Benthic, demersal, and pelagic components

\section{Relevant interventions}

- "Natural" blooms of ephemeral macroalgae and mats of macroalgae

- Manipulations of the abundance of an outbreaking macroalgal species (either direct manipulations or indirect manipulation made by changing nutrient levels, grazing intensity, etc.)

\section{Relevant comparators}

- Experimental comparisons of "treatment" (bloom present) and "control" (bloom absent) conditions

- Experimental and observational time series where the abundance of a bloom-forming species varies

- Comparisons of areas with and without blooms from observational studies

For the purposes of this review, we define macroalgal blooms as rapid, temporary, increases in macroalgal abundance. We will specifically search for studies of algal genera well-known to cause macroalgal blooms in temperate and subtropical waters, including Cladophora, Ulva (Enteromorpha), Ulvaria, Pylaiella (Pilayella; sometimes mistaken for Ectocarpus), Gracilaria, and Chaetomorpha. However, we will also include studies examining blooms or mats of other species.

\section{Relevant outcomes}

We will begin by searching for studies examining the effect of macroalgal outbreaks on a broad range of outcomes. The resources available to carry out this review are limited, so we may later decide to exclude studies examining some of these outcomes. This decision will be 
based on the number of studies found for different outcomes, not the apparent magnitude or direction of effects. Initially we will search for studies on:

1. Structure and diversity of communities or community components

- Species richness (numbers of species observed)

- Univariate diversity or evenness indices (Shannon's, Simpson's, Pielou's, etc)

- Abundance, biomass, density or cover of ecosystem components.

2. Ecosystem functions

- Productivity of the ecosystem or ecosystem components, measured as carbon fixation, respiration rate or other appropriate productivity measurements.

- Flows of energy or material between ecosystems or ecosystem components. For example, grazing or predation rates and measurements of ecosystem subsidies

- Biogeochemical cycling of nitrogen, phosphorus, carbon, oxygen, and sulphur, including both static and dynamic measures (e.g. static- nitrogen pool, dynamic- denitrification rate)

\section{Relevant study types}

- Manipulative experiments conducted in the field or laboratory

- Observational studies carried out in the field

We will consider studies from any time period. We will include grey literature and non-english studies if we find them following our general search strategy. However, we will not make additional, specific efforts to search for grey literature or non-english literature because we do not believe that we have adequate time or resources to do so in a thorough, unbiased, and systematic way. Non-significant results are often not published in scientific journals, but they might be more likely to appear in the grey literature. Our decision not to conduct grey-literature-specific searches could increase the chance that our results will be influenced by publication bias. Thus, we plan to evaluate and report on the potential effects of publication bias on our results as part of the review process.

Potential effect modifiers and reasons for heterogeneity We expect that the effects of macroalgal blooms may vary according to the following factors:

- Type of outcome (variable measured, ecosystem component, etc.)
- Type of ecosystem or habitat affected

- Features of blooms including species, size, magnitude, and duration

- Features of studies such as setting (lab vs. field), spatial scale, and timing of sampling (time since bloom started or ended)

- Region (i.e. different large marine ecosystems)

- Background ecosystem productivity

- Exposure (water movement, currents), temperature other physical or chemical parameters

\section{Study quality assessment}

Studies that meet the inclusion criteria will be assessed in terms of their susceptibility to bias, and the appropriateness of the study design in terms of estimating responses at the ecosystem scale. Each study will be classified along a number of dimensions:

a) Study setting - i) lab or ii) field

b) Study type - i) manipulative experiment or ii) observational

c) Appropriateness of controls- - i) appropriate ii) inappropriate iii) unclear

d) Replication - i) replicated or ii) unreplicated

e) Allocation of replicates - i) randomization ii) haphazard iii) other

f) Replication appropriateness - i) appropriate ii) inappropriate iii) unclear

g) Size of replicates - i) bay/bloom scale or larger ii) $>1 \mathrm{~m}^{2}$ iii) $<1 \mathrm{~m}^{2}$

h) Study extent - i) multiple blooms ii) full bloom iii) sub-bloom

i) Confounding factors - - i) present ii) not present iii) unclear

In addition to categorizing studies in this way, more specific details relating to each classification, such as the actual replicate size or the justification for considering replication appropriate or inappropriate, will be recorded and tabulated (See Additional file 1).

Determining whether controls are appropriate will be a subjective judgement, but the following criteria will be considered:

- do the controls appear to be spatially/temporally independent of the affected areas?

- are controls and affected areas sufficiently similar (consider, for example habitat and substrate type, degree of exposure, salinity, proximity to human activities).

If clear evidence is available in the paper that all of these criteria are met, then the controls should be considered valid, if there is evidence of failure to meet either 
of these criteria, the controls should be considered invalid and if it is not possible to make a proper assessment based on the information provided, then the study will be classified as 'unclear' in this regard.

If the authors refer to the replicates as being assigned randomly, or make reference to use of a random number table or random number generator, they will be classified as being randomly assigned. If the authors refer to the allocation as haphazard, or make reference to a procedure such as throwing a quadrat over their shoulder, allocation will be classified as 'haphazard'. All others methods will be classified as 'other', and described so that their susceptibility to bias can be assessed.

Determining whether or not replication has been carried out appropriately will be a subjective judgement, made after considering whether the replicates appear to be independent of one another in space and time (are they interspersed geographically, spread sufficiently in time, etc.). If the reviewer finds that the replicates are not independent, replication will be considered 'inappropriate'. If it is not possible to judge, the study will be classified as 'unclear'.

The dimensions of study quality will later be evaluated as potential effect modifiers in the meta-analysis. This will allow us to assess the influence of different aspects of study quality and design on the outcome of studies investigating macroalgal outbreaks. We are particularly interested in knowing whether lower quality studies are biased in a particular direction. Understanding this will strengthen our ability to make recommendations regarding the design of future studies. If particular dimensions of study quality are found to bias the outcome of studies, we will base our conclusions regarding the effects of macroalgal outbreaks on an analysis of studies classified in the category deemed to be of higher quality.

\section{Data extraction strategy}

In addition to being categorized in terms of aspects of study quality, studies that meet the inclusion criteria will be described in terms of their:

- Region (from US National Oceanic and Atmospheric Administration large marine ecosystem list)

- Geographic coordinates (field studies only)

- Dates (start and end dates, sampling dates)

- Habitat type (e.g. rocky subtidal, beach, open coast, lagoon)

- Aims/focus

- Study design (beyond those aspects covered in quality assessment)

- Response variables measured

Information on all of the outcome measures listed above will be extracted from relevant papers. Summary statistics (mean, standard deviation, standard error, confidence intervals) will be extracted from tables and graphs, using image analysis software when necessary. If raw data are provided rather than summary statistics, these will be extracted and the summary statistics will be calculated. Information on potential confounding variables or effect modifiers will also be extracted. Dates and location data may be used to obtain estimates of additional effect-modifying variables from other data sources (e.g. digital maps of sea surface temperature, primary productivity, potential solar radiation). If the required data cannot be extracted from the paper, the authors will be contacted and asked to provide it. A number of reviewers will independently extract data from different papers, but a subset of papers will be processed by all reviewers to verify that data extraction is consistent and repeatable.

\section{Data synthesis and presentation}

The review will first present the number and type of studies that cover the impact of blooms on each of the different outcome measures of interest (i.e. diversity, species richness, productivity of different parts of the ecosystem and nutrient cycling of different nutrients). Where sufficient data on the same outcome measure are available, a meta-analysis will be conducted. Where different measures of the same outcome can be meaningfully combined in a single effect, we will do so using standardised response measures (e.g. log response ratios). Initially, information from all studies of a given outcome will be combined, and a random-effects metaanalysis will be used to estimate effect sizes. Subgroup analysis or meta-regression will be conducted to assess the impact of study quality categories, different outcome measurements and other potential effect modifiers .

\section{Additional file}

Additional file 1: Critical appraisal and data extraction form.

Competing interests

The authors declare that they have no competing interests.

\section{Acknowledgements}

The authors wish to thank Christos Arvanitidis, Andrew Blight, Eva Chatzinikolaou, Tamar Guy-Haim, Paul Somerfield, and Ana Queiros for discussions that have influenced the content of this protocol, and for their help in carrying it out. The research leading to these results has received funding from the European Community's Seventh Framework Programme (FP7/2007-2013) under Grant Agreement No. 266445 for the project Vectors of Change in Oceans and Seas Marine Life, Impact on Economic Sectors (VECTORS), which is coordinated by Mel Austen.

\section{Author details}

${ }^{1}$ Lead reviewer, University College Dublin, Dublin, Ireland. ${ }^{2}$ Bangor University, Bangor, UK. ${ }^{3}$ University of Pisa, Pisa, Italy. ${ }^{4}$ Estonian Marine Institute, University of Tartu, Tallinn, Estonia. ${ }^{5}$ Israel Oceanographic and Limnological Research, Haifa, Israel. ' University College Dublin, Dublin, Ireland. 


\section{Authors' contributions}

DAL conceived of the study, coordinated development of the protocol, and drafted the manuscript. RCM, FB, JK, and GR participated in development of the protocol and drafting of the manuscript. TPC participated in conception of the study, development of the protocol, and drafting the manuscript. All authors read and approved the final manuscript.

Received: 9 February 2012 Accepted: 29 June 2012

Published: 29 June 2012

\section{References}

1. Halpern BS, Walbridge S, Selkoe KA, Kappel CV, Micheli F, D'Agrosa C, Bruno JF, Casey KS, Ebert C, Fox HE, Fujita R, Heinemann D, Lenihan HS, Madin EMP, Perry TM, Selig ER, Spalding M, Steneck R, Watson R: A global map of human impact on marine ecosystems. Science 2008, 319(5865):948-952.

2. Backer LC, McGillicuddy DJ: Harmful algal blooms at the interface between coastal oceanography and human health. Oceanography 2006, 19:94-106.

3. Condon RH, Steinberg DK, del Giorgio PA, Bouvier TC, Bronk DA, Graham WM, Ducklow HW: Jellyfish blooms result in a major microbial respiratory sink of carbon in marine systems. Proc Natl Acad Sci USA 2011, 108 (25):10225-10230.

4. Richardson AJ, Bakun A, Hays GC, Gibbons MJ: The jellyfish joyride: causes, consequences and management responses to a more gelatinous future. Trends Ecol Evol 2009, 24(6):312-322.

5. Valiela I, McClelland J, Hauxwell J, Behr PJ, Hersh D, Foreman K: Macroalgal blooms in shallow estuaries: Controls and ecophysiological and ecosystem consequences. Limnol Oceanogr 1997, 42(5):1105-1118.

6. Fletcher RL: The occurrence of "green tides"- a review. In Marine benthic vegetation:recent changes and the effects of eutrophication. Edited by Scharamm W, Nienhuis PH. Berlin: Springer; 1996:7-43.

7. Eriksson BK, Ljunggren L, Sandstrom A, Johansson G, Mattila J, Rubach A, Raberg S, Snickars M: Declines in predatory fish promote bloom-forming macroalgae. Ecol Appl 2009, 19(8):1975-1988.

8. Chrisafis A: Lethal algae take over beaches in northern France. In The Gaurdian. 2009. 10 August, 2009 edn.

9. Samuel H: Toxic seaweed on French coast sparks health fears. In The Telegraph. 2011. 22 July, 2011 edn.

10. Worm B, Lotze HK, Bostrom C, Engkvist R, Labanauskas V, Sommer U: Marine diversity shift linked to interactions among grazers, nutrients and propagule banks. Mar Ecol Prog Ser 1999, 185:309-314.

11. Hauxwell J, Cebrian J, Furlong C, Valiela I: Macroalgal canopies contribute to eelgrass (Zostera marina) decline in temperate estuarine ecosystems. Ecology 2001, 82(4):1007-1022.

12. Wharfe JR: Ecological survey of benthic invertebrate Macrofauna of Lower Medway Estuary, Kent. J Anim Ecol 1977, 46(1):93-113.

13. Everett RA: Intertidal distribution of infauna in a Central California Lagoon - the role of seasonal blooms of macroalgae. J Exp Mar Biol Ecol 1991, 150(2):223-247.

14. Franz DR, Friedman I: Effects of a macroalgal mat (Ulva lactuca) on estuarine sand flat copepods: an experimental study. J Exp Mar Biol Ecol 2002, 271(2):209-226.

15. Jones M, Pinn E: The impact of a macroalgal mat on benthic biodiversity in Poole Harbour. Mar Poll Bull 2006, 53(1-4):63-71.

16. Wilson KA, Able KW, Heck KL: Predation rates on Juvenile blue crabs in estuarine nursery habitats - evidence for the importance of macroalgae (Ulva-Lactuca). Mar Ecol Prog Ser 1990, 58(3):243-251.

17. Holmquist JG: Benthic macroalgae as a dispersal mechanism for fauna - influence of a marine tumbleweed. J Exp Mar Biol Ecol 1994, 180 (2):235-251.

18. Holmquist JG: Disturbance and gap formation in a marine benthic mosaic: influence of shifting macroalgal patches on seagrass structure and mobile invertebrates. Mar Ecol Prog Ser 1997, 158:121-130.

19. Dolbeth M, Pardal MA, Lillebo Al, Azeiteiro U, Marques JC: Short- and long-term effects of eutrophication on the secondary production of an intertidal macrobenthic community. Mar Biol 2003, 143(6):1229-1238.

20. Macia S: The effects of sea urchin grazing and drift algal blooms on a subtropical seagrass bed community. J Exp Mar Biol Ecol 2000, 246(1): 53-67.
21. Thybo-Christesen M, Rasmussen MB, Blackburn TH: Nutrient fluxes and growth of cladophora-sericea in a shallow Danish Bay. Mar Ecol Prog Ser 1993, 100(3):273-281.

22. Hardison AK, Canuel EA, Anderson IC, Veuger B: Fate of macroalgae in benthic systems: carbon and nitrogen cycling within the microbial community. Mar Ecol Prog Ser 2010, 414:41-55.

23. Hughes TP: Catastrophes, phase-shifts, and large-scale degradation of a Caribbean coral-reef. Science 1994, 265(5178):1547-1551.

24. McCook LJ, Jompa J, Diaz-Pulido G: Competition between corals and algae on coral reefs: a review of evidence and mechanisms. Coral Reefs 2001, 19(4):400-417.

25. McManus JW, Polsenberg JF: Coral-algal phase shifts on coral reefs: ecological and environmental aspects. Prog Oceanogr 2004, 60(2-4): 263-279.

26. Bruno JF, Sweatman H, Precht WF, Selig ER, Schutte VGW: Assessing evidence of phase shifts from coral to macroalgal dominance on coral reefs. Ecology 2009, 90(6):1478-1484.

doi:10.1186/2047-2382-1-7

Cite this article as: Lyons et al:: What are the effects of macroalgal blooms on the structure and functioning of marine ecosystems? A systematic review protocol. Environmental Evidence 2012 1:7.

\section{Submit your next manuscript to BioMed Central and take full advantage of:}

- Convenient online submission

- Thorough peer review

- No space constraints or color figure charges

- Immediate publication on acceptance

- Inclusion in PubMed, CAS, Scopus and Google Scholar

- Research which is freely available for redistribution 